\title{
Multitude Regional Texture Extraction for Image Segmentation of Aerial and Natural Images
}

\section{S. Rizwana}

\begin{abstract}
Image processing plays a major role in evaluation of images in many concerns. Manual interpretation of the image is time consuming process and it is susceptible to human errors. Computer assisted approaches for analyzing the images have increased in latest evolution of image processing. Also it has highlighted its performance more in the field of medical sciences. Many techniques are available for the involvement in processing of images, evaluation, extraction etc. The main goal of image segmentation is cluster pixeling the regions corresponding to individual surfaces, objects, or natural parts of objects and to simplify and/or change the representation of an image into something that is more meaningful and easier to analyze.

The proposed method is to conquer segmentation and texture extraction with Regional and Multitude and techniques involved in it. First for aerial and natural imaging we present region based segmentation. Homogeneous regions depend on image granularity features. Second a local threshold based multitude texture regional seed segmentation for Aerial and natural image segmentation is proposed. Here extraction is done with dimensions comparable to the speckle size are to be extracted. The algorithm provides a less natural metrics awareness in a minimum user interaction environment. The shape and size of the growing regions depend on look up table entries. The experimental evaluation is conducted with training samples of natural and aerial images to show the performance of multitude textural extraction for more efficient image segmentation with sharp demarcation of edge portions along with intensity levels.
\end{abstract}

Key Words: Image Segmentation, Texture Extraction, Region Growing, Natural and Aerial Images

\section{Introduction}

In Image processing the inputs is mainly an image photograph or video frame. The output after image processing will be either an image or set of characteristics of parameters related to the image. Image segmentation is one of the important aspects in processing of image. Its work is clustering the pixels and changes the representation into some meaningful images. Image segmentation is typically used to locate objects and boundaries (lines, curves, etc.) in images. This usually results in set of segments that collectively cover the entire image.

There are many types of image segmentation available here. Some of them are Edge detection; Region boundaries, Region Growing, Threshold etc., Region boundaries and edges are closely related. So this has been used as the bade of another segmentation technique. If no edges are detected, the neighboring pixels are examined and added to a region class. This process is iterated for each boundary pixel in the region. If adjacent regions are found, a region-merging algorithm is used on weak edges are dissolved and strong edges are left in tact.

The major advantage of Region growing over conventional segmentation is the borders of regions found by region growing are perfectly thin and connected. And also the algorithm is very stable with respect to noise. The important thing is that membership in a region can be based on multiple criteria. We can take advantage of several image properties, such as low gradient or gray level intensity value, at once.

\section{Literature Works}

In image segmentation we have a simple method called thresholding. The method is based on a cliplevel (or a threshold value) to turn a gray-scale image into a binary image.The key of this method is to select the threshold value (or values when multiple-levels are selected).

In image processing we have a well-developed field called Edge detection. There is a sharp adjustment in intensity in region boundaries so they are closely related. Edge detection techniques have been the base of all segmentation technique. The edges identified by edge detection are often disconnected. Closed region boundaries are necessary to segment an object from an image.

Region Growing is an approach to image segmentation in which neighboring pixels are examined and added to a region class if no edges are detected. This process is iterated for each boundary pixel in the region. If adjacent regions are found, a region-merging algorithm is used on weak edges are dissolved and strong edges are left in tact.

Region Growing offers several advantages over conventional segmentation techniques. Unlike gradient and Laplacian methods, the borders of regions found by region growing are perfectly thin (since we only add 
pixels to the exterior of our region) and connected. The algorithm is also very stable with respect to noise. Our region will never contain too much of the background, so long as the parameters are defined correctly. Other techniques that produce connected edges, like boundary tracking, are very unstable. Most importantly, membership in a region can be based on multiple criteria. We can take advantage of several image properties, such as low gradient or gray level intensity value, at once. Hybrid method uses both boundary based and region growing method.

\section{Region based Texture Extraction Method}

Segmentation is usually a process of partitioning a digital image into multiple segments the major objective of segmentation is to simplify and/or change the representation of an image into something that is more meaningful and easier to analyze. . Image segmentation is typically used to locate objects and boundaries (lines, curves, etc.) in images. In short we can say, image segmentation is the process of assigning a label to every pixel in an image such that pixels with the same label share certain visual characteristics.

As said earlier Region Growing offers several advantages over conventional segmentation techniques. Here the borders of regions are perfectly thin and connected. The algorithm is very stable with respect to noise. Until the parameters are defined correctly the region never contains too much of background. Most importantly, membership in a region can be based on multiple criteria. The image properties such as low gradient or gray level intensity value are also taken into account.

The imaging is non-ionizing, fast, portable, inexpensive and capable of real time imaging. The images typically contain significant speckle and other artifacts which complicate image interpretation and automatic processing. The speckle formation is one in which the images of relatively poor quality and analysis in general is described. The speckles mask the low contrast lesion and reduce the ability of radiologist to resolve the actual information. It is difficult to segment the ultrasound image to detect objects with the correct position and shape because of speckle formation. In addition, boundary edges are usually incomplete, missing or weak at some places. For the aerial and natural image segmentation, mostly the methods are focused on five main approaches, namely, threshold technique, boundary-based method, region based methods, hybrid techniques which combine boundary and region criteria and active contour based approach.

Threshold is a technique where decision is based on local pixel information. This is an effective method when the intensity levels of the objects fall squarely outside the range of levels in the background. In Edge - based methods the weakness in connecting together broken contour lines make them, too, prone to failure in the presence of blurring. In active contour model, the main idea is to start with some initial boundary shape represented in the form of spline curves and iteratively modifies it by applying various shrink/expansion operations according to some energy function.

The Region - based texture extraction method usually done by partitioning the image into connected regions by grouping neighboring pixels of similar intensity levels. Then adjacent regions are merged. Over stringent criteria create fragmentation; lenient ones overlook blurred boundaries and over merge. Hybrid techniques using a mix of the methods above are also popular.

In our paper, a local threshold based multitude texture regional seed segmentation for medical image segmentation is proposed. Here, for local statistics, lookup table is prepared to use in initial region. Then pixels are grouped that satisfy a specify homogeneity criteria. Finally it produces a homogeneous region, and merging the neighboring regions, which have similar intensity values.

Algorithm implementation is done by using seeded region growing procedure where each pixel is taken as seed point. The shape and size of the growing regions depend on look up table entries. The region merging is done after the region growing. This suppresses the high frequency artifacts. The merged regions output will be in the form of segmented image with higher efficiency than watershed method. The less noise sensitive pixel is produced in SRG algorithm. It also allows a segmentation of the accurate homogeneous regions compared with morphological filter segmentation.

\section{Multitude regional image segmentation}

The next proposal is applying texture feature-based multitude regional image segmentation on aerial and natural image. Less metrics awareness in a minimum user interaction environment is provided in the proposed system. This helps in batch work and to the novice computer users. The usage of 2D semi-automatic SRG with texture feature is deployed in aerial and natural image segmentation. Feasible texture feature in aerial and natural image segmentation are found after comparing with morphological filter and watershed methods

For aerial and natural image local threshold selection based segmentation procedure has been developed. Look up table controls the preserved aerial and natural images that consist of homogeneity and statistical values of each pixel. $11 \times 11$ window size is used for computation of local statistics. This choice is based on the small homogeneous regions, which are produced by the granularity. The window size must be large enough for the measurement of homogeneity region criteria and statistical similarity bound. The parameter selection of the similarity bound depends on the granularity or speckle into the images. The initial growing 
region shows the large number of false homogeneous region into the image, which was joined with their neighboring region by merging. The parameters for merging criteria depend on the high frequency artifacts such as over segmentation. This algorithm can be used for fully developed speckle images with efficient segmentation. The merged regions reduce over segmentation without using further smoothing into the image. The final segmentation results exhibit accurate homogeneous regions without implementing texture-based analysis.

\subsection{Architecture}

The diagram 4.1 given below illustrates how the image is segmented provided with the input training images. When trained with input data from image, intensity ranges and RGB and an alpha value is applied as input to the features extracted from the training images. The color-corrected RGB image is then converted to image features which are more suitable for determining the borders between regions. For intensity ranges some of the popular approaches are the threshold techniques, edge-based methods, region-based techniques and connectivity-preserving methods are adopted. Our multitude region-based method usually works as follows: the training image is further divided into regions by way of grouping the neighboring pixels that has the similar intensity ranges. The regions that are adjacent to the pixels are then merged based on some criterion such as the RGB value or the alpha distribution value obtained. Texture extraction is the identification of regions based on their texture. We have also shown that the texture extracted can also be used for certain tasks such as region segregation and region outliers. The texture extracted in applied for multitude of texture pixels with the function Multi Texture. The resultant image obtained is the segmented image.

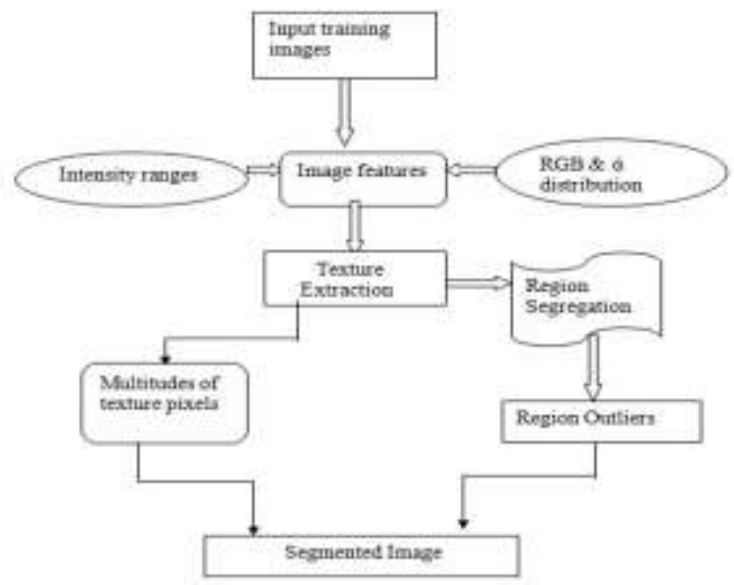

Fig1. Architecture of segmented image

\subsection{Formulation of Multitude Textual Extraction Image Features}

Consider a finite training set of images $\mathrm{S}=\{\mathrm{s} 1, \mathrm{~s} 2, \ldots \mathrm{sn}\}$ and can be represented by a set $\mathrm{D}$.

$$
\sum_{i=1}(1 / 2|\mathrm{si}+\mathrm{D} \alpha \dot{\mathrm{i}}|+\lambda(\mathrm{RGB}) \mathrm{i}
$$

Where $\lambda$ denotes the intensity ranges and ói denotes the alpha distribution and (RGB)i gives the RGB value for a single image

\section{Texture Extraction}

Texture extraction is done in two steps using the set $\mathrm{D}$ and summing it up. It is learned using $\mathrm{S}=\{\mathrm{s} 1$,

$\mathrm{s} 2, \ldots, \mathrm{sn}\}$. Where $\mathrm{Si}, \mathrm{i}=1,2 \ldots \mathrm{m}$ are the patches extracted with size $\mathrm{d} * \mathrm{~d}$. from texture images in training set.

Sum up for all the coefficients for particular texture

$$
\mathrm{T}(\mathrm{S})=\Sigma_{\mathrm{i}=1,}{ }^{\mathrm{n}}(\text { ói })
$$

\section{Region Outlier \& Segregation}

Outlier rate $=(\mathrm{x} / \mathrm{n}) * 100$

Where $\mathrm{x}$ denotes the number of outliers found and $\mathrm{n}$ denotes the total number of training images.

\section{Multitudes of Texture Pixels}

Texture coordinate Cá between two pixels $\mathrm{C} 0$ and $\mathrm{C} 1$ is given by

$$
\mathrm{C} \alpha \dot{\alpha}=(1+\dot{\alpha}) \mathrm{C} 0+\dot{\alpha} \mathrm{C} 1
$$

\subsection{ALGORITHM}

Algorithm Multitude Regional Texture Extraction for Natural Image Segmentation 
Input a finite set of training images

Assignment of the set denote D

Loop

For $\mathrm{S}=\{\mathrm{s} 1, \mathrm{~s} 2, \ldots \mathrm{sn}\}$ where $\mathrm{i}=1,2, \ldots \mathrm{n}$

Perform $\Sigma(1 / 2 \mid$ si + D ói $\mid+\lambda(\mathrm{RGB}) \mathrm{i}$

Exit

Evaluate Texture extraction

For $\mathrm{S}=\{\mathrm{s} 1, \mathrm{~s} 2, \ldots \mathrm{sn}\}$ where $\mathrm{I}=1,2, . . \mathrm{n}$

Perform $\mathrm{d} * \mathrm{~d}$

Exit

Perform $\mathrm{T}(\mathrm{S})=\sum_{\mathrm{i}=1,}{ }^{\mathrm{n}}$ ( ái )

Evaluate Segmented Image

Perform Outlier rate $=(\mathrm{x} / \mathrm{n}) * 100$

Exit

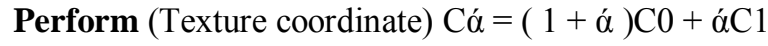

End loop

\section{Experimental Results on Multitude RegionalTexture Extraction}

The experimentation conducted on Natural images to evaluate the performance of proposed local phase and threshold texture extraction for future Natural segmentation. Implementation of the proposed algorithm is done in MATLAB. In addition to noise removal, the proposed model also present qualitative results for the texture extraction of the natural image edges. The localization accuracy of natural surface detection technique and assessing the accuracy of measuring relative inner layers of separation is a clinically relevant task for which the system uses 2D imaging. The screen shots of the natural in this proposed technique is shown in figure given below.

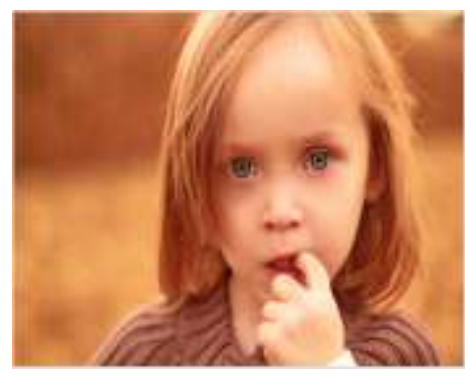

(a) Input Image

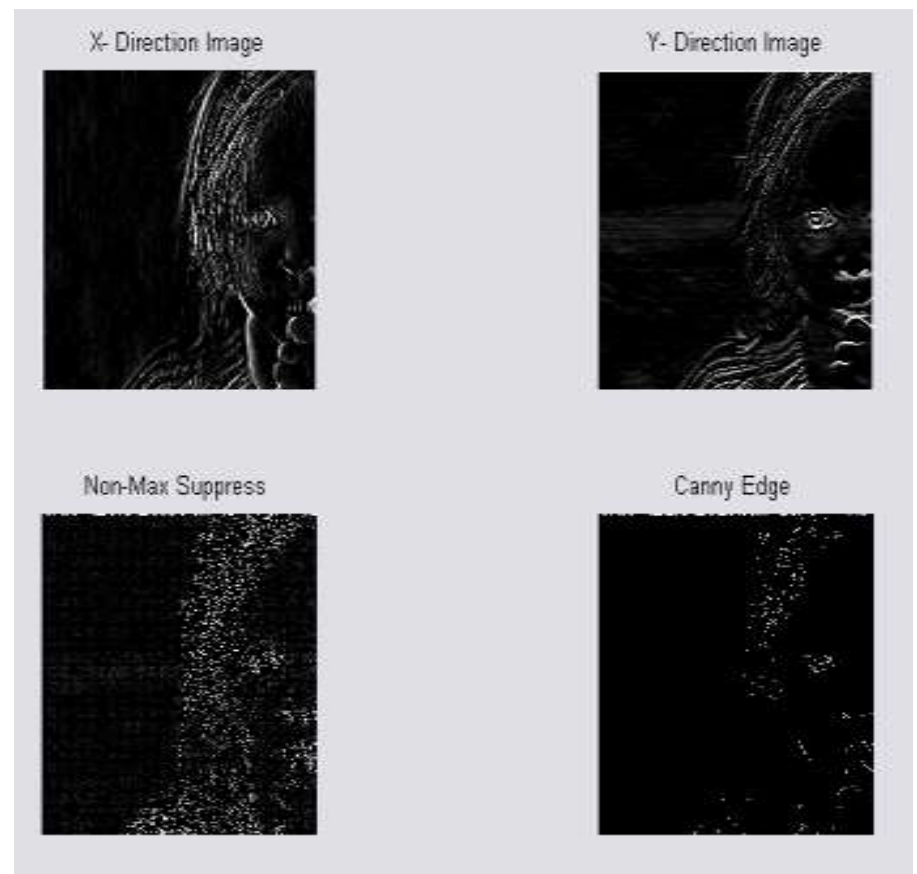

(b) Edge Detected Image 


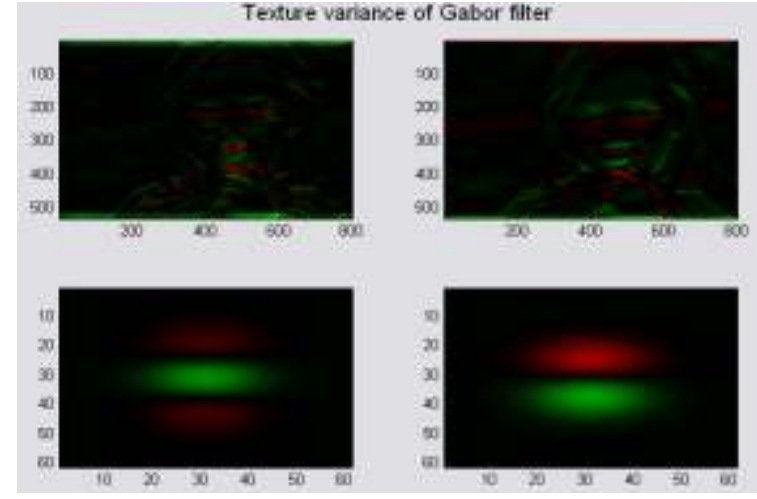

(c) Texture Image

The datasets used here are segmented homogeneous region, number of images and number of pixels in segmented region. The experimentation presented here gives a specify homogeneity criteria and produce the homogeneous region, and merging the neighboring regions, which have similar intensity values.

This can be especially helpful for batch work or to novice computer users. The deployment of aerial and natural image segmentation is used as a 2D semi-automatic SRG with texture feature. They are compared with the morphological filter (12\% less homogeneous regions) and watershed methods (15\% less homogeneous pixels) and found to be a feasible texture feature in natural image segmentation.

Seeded region growing procedure algorithm is implemented where each pixel is taken as seed point. The shape and size of the growing regions depend on look up table entries. Look up table also consists of homogeneity and statistical values of each pixel. The less noise sensitive pixel is produced in SRG algorithm. 11 $\mathrm{x} 11$ window size is used for computation of local statistics. The merged regions reduce over segmentation. Further smoothing into the image is not needed here. The final segmentation results exhibit accurate homogeneous regions without implementing texture-based analysis

The performance of the datasets that are compared with the existing system with segmented homogenous region and normal segmented region of threshold technique is given below.

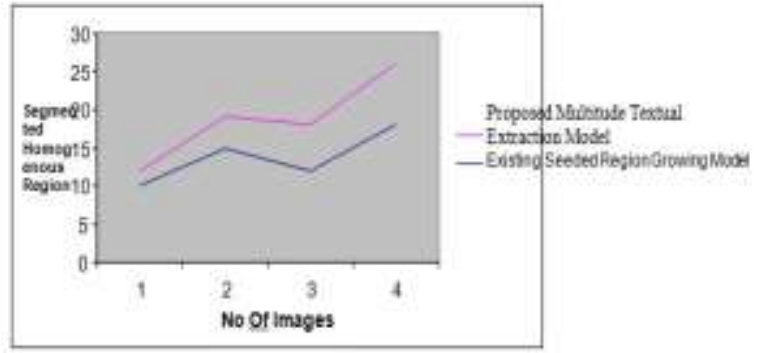

(a)

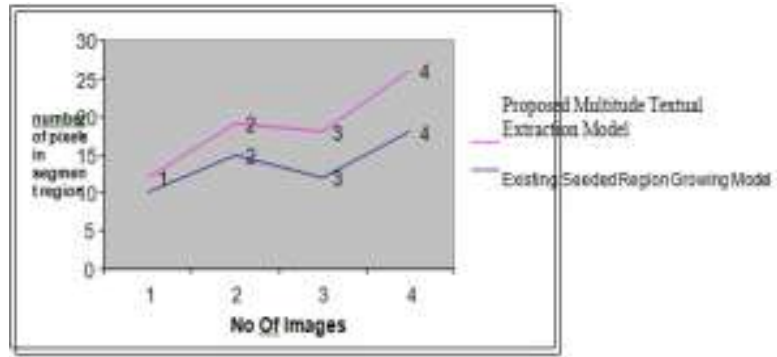

(b)

VI.

Conclusion:

In threshold technique, because spatial information is ignored, however, blurred region boundaries can create havoc. In Edge-based methods there is a weakness in connecting together broken contour lines make them, too, prone to failure in the presence of blurring. The region merging which is done after the region growing suppresses the high frequency artifacts. The region that is merged output will be in the form of segmented image with higher efficiency than watershed method. In region based methods. Over stringent criteria of proposed multitude texture extraction model for image segmentation create fragmentation, overlook blurred boundaries and over merge. 
Aerial and natural imaging, the resulting contours after image segmentation can be used to create $3 \mathrm{D}$ reconstructions with the help of interpolation algorithms. The algorithm developed for fully speckle images with efficient segmentation to show sharp and fine edges of the segmented portion of the images. The merged regions reduce over segmentation without using further smoothing into the image.

Segmented edge detection is made with multitudes of pixels on its image portions to arrive more finite demarcation between pixel variance on any given image. Multitudes of textural region boundaries and edges are closely related, since there is often a sharp adjustment in intensity at the region boundaries. Multitudes of pixels in the region growing offer several advantages over conventional segmentation technique which in turn an accurate homogeneous regions of segments are identified with texture based analysis is resulted.

\section{References}

[1] T.-Y. Law and P. A. Heng, (2000) "Automated extraction of bronchus from 3-D CT images of lung based on genetic algorithm and 3-D region growing”, Proc. SPIE 3979, Medical Imaging 2000: Image Processing, 906-916.

[2] R. Susomboon, D. S. Raicu, and J. D. Furst, (2006)“Pixel-Based Texture Classification of Tissues in Computed Tomography", CTI Research Symposium, Chicago, April 2006.

[3] J. E. Koss, F. D. Newman, T. K. Johnson, D. L. Kirch, (1999) "Abdominal organ segmentation using texture transform and a Hopfield neural network", IEEE Trans. Medical Imaging, Vol.18, 640-648.

[4] R. Adams, L Bischof, (1994) "Seeded region growing". IEEE Transaction Pattern Analysis Machine Intelligency 16, 641 -647.

[5] N. A. Mat-Isa, M. Y. Mashor \& N. H. Othman, (2005) "Seeded Region Growing Features Extraction Algorithm; Its Potential Use in Improving Screening for Cervical Cancer". International Journal of the Computer, the Internet and Management (ISSN No: 08587027) Vol. 13. No. 1 January-April

[6] Y. Tuduki, K. Murase, M. Izumida, H. Miki, K. Kikuchi, K. Murakami \& J. Ikezoe (2000). “Automated Seeded Region Growing Algorithm for Extraction of Cerebral Blood Vessels from Magnetic Resonance Angiographic Data" Proceedings of The 22nd Annual International Conference of the IEEE Engineering in Medicine and Biology Society 1756-1759

[7] P. A. Venkatachalam, U. K.Ngah, A. F. M. Hani\& A. Y. M. Shakaff, (2002). "Seed Based Region Growing Technique in Breast Cancer Detection and Embedded Expert System". Proceedings of International Conference on Artificial Intelligence in Engineering and Technology 464-469

[8] V. A. Kovalev, F. Kruggel, H.-J Gertz, and D.Y. von Cramon. (2001) "Three-dimensional texture analysis of MRI brain datasets" IEEE Trans. on Medical Imaging, 20(5): 424-433.

[9] S. A. Karkanis, et al., (1999) "Detecting abnormalities in colonoscopic images by texture descriptors and neural networks," Proc. of the Workshop Machine Learning in Med. App., 59-62.

[10] A. Madabhushi, M. Feldman, D. Metaxas, D. Chute, and J. Tomaszewski. (2003) "A novel stochastic combination of 3D texture features for automated segmentation of prostatic adenocarcinoma from high resolution MRI." Medical Image Computing and Computer-Assisted Intervention, volume 2878 of Lecture Notes in 8 J. Wu et al. / J. Biomedical Science and Engineering 2 (2009) 1-8 Computer Science, pp. 581-591. Springer-Verlag

[11] B. W. Whitney, N. J. Backman, J. D. Furst, D. S. Raicu, (2006) "Single click volumetric segmentation of abdominal organs in Computed Tomography images”, Proceedings of SPIE Medical Imaging Conference, San Diego, CA, Februar.

[12] J. Wu, S. Poehlman, M. D. Noseworthy, M. Kamath, (2008) Texture Feature based Automated Seeded Region Growing in Abdominal MRI Segmentation, 2008 International Conference on Biomedical Engineering and Informatics, Sanya, China, May 2730 . 